\title{
The reproducibility and validity verification for body composition measuring devices using bioelectrical impedance analysis in Korean adults
}

\author{
Seung-Won Yang ${ }^{1}$, Tai-Hyung Kim² ${ }^{2}$ Hyun-Min Choi ${ }^{3, *}$ \\ 'Department of Physical Education, Baekseok University, Cheonan, Korea \\ ${ }^{2}$ Graduate School of Technology Management, Kyung Hee University, Yongin, Korea \\ ${ }^{3}$ Department of Sports Leisure, Gwangju University, Gwangju, Korea
}

The purpose of this study is to examine the validity and reproducibility of impedance body fat measurement devices measuring the body composition of Korean male and female adults using three bioelectrical impedance analyzers. We compared two methods for evaluating body composition: dual energy X-ray absorptiometry (DEXA), and bioelectrical impedance analysis (BIA). Subjects were 200 healthy adult Korean males and females whose mean \pm standard deviation (range) age, standing height, body weight, and body mass index (BMI) were $44.1 \pm 14.5$ years, $172.8 \pm 7.4 \mathrm{~cm}, 76.0 \pm 12.8 \mathrm{~kg}$, and $25.4 \pm 3.3 \mathrm{~kg} / \mathrm{m}^{2}$, and $44.5 \pm 14.7$ years, $158.7 \pm 5.8 \mathrm{~cm}, 58.3 \pm 8.3 \mathrm{~kg}$, and $23.2 \pm 3.0 \mathrm{~kg} / \mathrm{m}^{2}$, respectively. As a result, first of all, the reproducibility of the bioelectrical impedance analyzer had very high coefficients at $r=0.998, r=0.997$ between men and women, respectively. The correlation coefficients among three comparisons for lean body mass (LBM) were provided the following coefficients: $r=0.951$ for DEXA vs. ACCUNIQ BC720, $r=0.950$ for DEXA vs. ACCUNIO BC360, and $r=0.946$ for DEXA vs. ACCUNIO $\mathrm{BC} 380$ in men. In the results for women, they also had the very high following coefficients: $r=0.956$ for DEXA vs. ACCUNIQ BC720, $r=0.946$ for DEXA vs. ACCUNIO BC360, and $r=0.957$ for DEXA vs. ACCUNIO BC380 in LBM. In conclusion, this research showed a higher correlation in terms of accuracy compared to existing BIA-based body composition measurement techniques, and the accuracy of LBM was improved with high correlation coefficients through the algorithm that was improved using the multifrequency BIA method in the ACCUNIO BC products.

Keywords: Validity, Reproducibility, Body composition, Adults, Bioelectrical impedance analysis

\section{INTRODUCTION}

Interests in body composition evaluation have increased in sectors including physiology, nutritional science, sports science, and measurement and evaluation in physical activity. Although various approaches are used for evaluation, there are some difficulties in terms of the validity to accomplish different research purposes. The measurement tools to evaluate body composition include air displacement plethysmography (BODPOD) (Fields et al., 2000), dual energy X-ray absorptiometry (DEXA) (Haarbo et al., 1991), computed tomography (CT), magnetic resonance imaging (MRI) (Pelizzari et al., 1989), and ultra sound (Saarelainen et al., 2007).
Even though the reliability or validity of these are accurate when it comes to lab tests, they are expensive, require research subjects to visit specific institutions, and need professional and trained testers due to the complicated and highly sensitive characteristics.

In contrast, the underwater weighing technique, which is widely used in different labs, is inexpensive, but it requires a large space and is difficult to apply for children, disabled patients or seniors. Against this background, body composition devices using bioelectrical impedance analysis (BIA) have recently been on the market and used at sites related to health and physical strength care or home. The noninvasive tool, BIA, evaluates body composition without complex technology at lower costs and time. There-

\footnotetext{
${ }^{*}$ Corresponding author: Hyun-Min Choi (D) https://orcid.org/0000-0003-1825-8408 Department of Sports Leisure, Gwangju University, 277 Hyodeok-ro, Nam-gu, Gwangju 61743, Korea

Tel: +82-62-670-2426, Fax: +82-62-670-2218, E-mail: hmchoi@gwangju.ac.kr Received: May 29, 2018 / Accepted: July 20, 2018
}

This is an Open Access article distributed under the terms of the Creative Commons Attribution Non-Commercial License (http://creativecommons.org/licenses/by-nc/4.0/) which permits unrestricted non-commercial use, distribution, and reproduction in any medium, provided the original work is properly cited. 
fore, many researchers have reported that body composition evaluation using BIA has high validity (Nakadomo et al., 1990). On the other hand, it also has validity issues of the included estimation equation when applied to different races, healthy and strong athletes, seniors, and patients. Therefore, the validity of a number of BIA estimation equations has been studied (Stolarczyk et al., 1994; Tanaka et al., 1992). Measuring devices using BIA have been developed and commercialized at home and abroad in forms for which adsorption electrodes or gel are not required. In Korea, starting from the development of BIA measurement devices in which hands and feet are contacted to electrodes and measurements are conducted in a standing position, such as Inbody 3.0 (Biospace Co., Seoul, Korea) and X-scan Plus (Jawon Medical Co., Daejeon, Korea). Recently, multifrequency BIA measuring devices for which mobile applications were applied to make the measurement practical in daily life and easy for healthy and strong athletes or seniors including ACCUNIQ BC720, ACCUNIQ BC360, and ACCUNIQ BC380 (SELVAS Healthcare Inc., Daejeon, Korea), have been introduced.

Due to the different included estimation equations, which were above mentioned as a problem of BIA, or electrode contacts, the newly developed body composition measuring devices should be verified for validity for accurate measurement of body composition. The references of body composition measurement include the most widely used underwater weighing, BODPOD, that measure whole body mass to estimate density (Katch et al., 1989), and DEXA, which is known as an X-ray method. As for DEXA, the error for repeated measurement is within $1 \%$, which makes it more reliable and precise than the underwater weighing technique with a repeated measurement error of $2.7 \%-3.7 \%$ (Kohrt, 1998). Moreover, it was reported that the underwater weighing technique and DEXA had similar accuracy compared to other measuring devices for Asians to evaluate body composition (Kitano et al., 2001; WHO Expert Consultation, 2004).

Thus, this research aims to study the reproducibility and validity of new Korean body composition measuring devices, including ACCUNIQ BC720, ACCUNIQ BC360, and ACCUNIQ BC380 based on the reference of DEXA, which is very convenient and stable as it tests research subjects in a lying position.

\section{MATERIALS AND METHODS}

\section{Survey subjects}

For the research, 200 adults aged between 20 and $69(97$ men and 103 women) who live in Gyeonggi province, are not orthope-
Table 1. Descriptive characteristics of subjects

\begin{tabular}{lcc}
\hline Variables & Men $(\mathrm{n}=97)$ & Women $(\mathrm{n}=103)$ \\
\hline Age $(\mathrm{yr})$ & $44.1 \pm 14.5$ & $44.5 \pm 14.7$ \\
Height $(\mathrm{cm})$ & $172.8 \pm 7.4$ & $158.7 \pm 5.8^{*}$ \\
Weight $(\mathrm{kg})$ & $76.0 \pm 12.8$ & $58.3 \pm 8.3^{*}$ \\
Body mass index $\left(\mathrm{kg} / \mathrm{m}^{2}\right)$ & $25.4 \pm 3.3$ & $23.2 \pm 3.0^{*}$ \\
DEXA PBF $(\%)$ & $22.0 \pm 5.1$ & $31.0 \pm 5.3^{*}$ \\
DEXA LBM $(\mathrm{kg})$ & $57.9 \pm 7.8$ & $39.3 \pm 4.7^{*}$ \\
\hline
\end{tabular}

Values are presented as mean \pm standard deviation.

DEXA PBF, percent body fat by using dual energy X-ray absorptiometry; DEXA LBM, lean body mass by using dual energy $X$-ray absorptiometry.

${ }^{*} P<0.05$, significant difference.

dically or psychologically abnormal, and have no disabilities were recruited. They consist of 40 research subjects from each age group of the 20s, 30s, 40s, 50s, and 60s, and healthy and strong athletes were also included. The participating research subjects were determined based on preceding research related to this research. Therefore, this research recruited a total of 200 research subjects, 40 per age group. Their human body characteristics are shown in Table 1.

\section{Experimental procedure}

This research analyzed the validity for the reference developed by an Hologic QDR-4500W fan-beam DEXA scanner (Hologic Inc., Bedford, MA, USA), and through body composition measuring devices ACCUNIQ BC720, ACCUNIQ BC360, ACCUNIQ BC380 (SELVAS Healthcare Inc.) newly developed for healthy and strong athletes as well as people in various age groups.

The research subjects were made to refrain from high intensity exercise and have a good sleep $48 \mathrm{hr}$ before the measurement. They were also prohibited from drinking $24 \mathrm{hr}$ before the test, eating or drinking any beverage $4 \mathrm{hr}$ before the measurement, and running at the lab. On the day of measurement, they were fully informed of the purpose, items, procedure and precautions of the test, signed the health history questionnaire and informed consent, rested for more than $30 \mathrm{~min}$ in a comfortable position, removed any metal accessories and belts, and put on light clothing to participate in the measurement. All procedures were reviewed and approved by the World Association of Public Health and Beauty Institutional Review Board (1-20170113119-AB-N-01-04).

\section{Survey tool}

\section{Measurement of standing height and body weight}

Height was measured to the nearest $0.1 \mathrm{~cm}$ using the Martin human body measuring system, and body weight was automatically recorded with a measuring device using BIA. Based on the 
measured height and body weight, body mass index (BMI) was calculated.

\section{Measurement using DEXA}

With the reference of DEXA, the body composition measurement was conducted at the Human Anatomy and Physiology Lab of Kyung Hee University. The research subjects who arrived at the lab removed all metal materials, changed to a lab dress, entered their basic data (name, nationality, height, body weight, age, date of birth, etc.) on the computer program, lay on the measurement board in an anatomical position, and were measured for about 5-6 min. Upon the measurement, their data were automatically printed out of the computer to be used for the validity analysis with references of lean body mass (LBM) and percent body fat (PBF).

\section{Measurement using BIA}

The body composition measurement was conducted with models applying the principle of multifrequency including ACCUNIQ BC720, ACCUNIQ BC360 and ACCUNIQ BC380 that have been developed and used in Korea. The subjects' palms and soles were cleaned with electrolyte tissues before stepping on the foothold electrodes of each measuring device; their ID, height, age, and gender were input, and body weight was automatically measured. For the measurement, they stood up in an anatomical position with their arms open and away from their body at about a $30^{\circ}$ angle; such position was maintained until the end of the measurement, and they were prohibited from talking or moving during the process, if not necessary. Each measurement took about 2 min, respectively. Among the collected items, LBM and PBF were utilized for the validity analysis.

\section{Data analyses}

All measured data were analyzed as follows using Sigma Stat

Table 2. Reproducibility verification of PBF measured by bioelectrical impedance analyzer

\begin{tabular}{|c|c|c|c|c|c|c|}
\hline & \multicolumn{2}{|c|}{$\operatorname{Men}(n=40)$} & \multicolumn{2}{|c|}{ Women $(n=40)$} & \multicolumn{2}{|c|}{ Total $(n=80)$} \\
\hline & $\operatorname{PBF}(\%)$ & $r$ & $\operatorname{PBF}(\%)$ & $r$ & $\operatorname{PBF}(\%)$ & $r$ \\
\hline Trial & & 0.996 & & 0.998 & & 0.997 \\
\hline $1 \mathrm{st}$ & $18.8 \pm 5.9$ & & $31.0 \pm 4.4$ & & $26.8 \pm 6.8$ & \\
\hline 2nd & $18.9 \pm 5.9$ & & $31.1 \pm 4.4$ & & $26.9 \pm 6.7$ & \\
\hline $3 r d$ & $18.9 \pm 5.9$ & & $31.1 \pm 4.4$ & & $26.9 \pm 6.7$ & \\
\hline
\end{tabular}

Values are presented as mean \pm standard deviation.

The intraclass correlation coefficient ( $h$ ) were calculated from three trial measurements by the same examiner during the same test session.

PBF, percent body fat. version 11.2 (Systat Software Inc., Richmond, CA, USA) according to the analysis purpose. For the differences among the human physical characteristics and measurement variables by gender, descriptive statistics and an independent $t$-test were performed. As for the validity, correlation analysis (Pearson correlation) and liner regression analysis were carried out with data measured with DEXA and BIA, and correlation and regression equations were calculated. Moreover, intraclass correlation coefficient (ICC) analysis was conducted through twice repeated measurements to investigate the reproducibility of measuring devices using BIA. All statistically significant levels were bilaterality and it was determined significant when $P<0.05$.

\section{RESULTS}

\section{The characteristics of the research subjects}

The mean age of the research subjects was $44.1 \pm 14.5$ for men and $44.5 \pm 14.7$ for women. In PBF measured by DEXA, it was $22.0 \% \pm 5.1 \%$ for male subjects and for $31.0 \% \pm 5.3 \%$ females, while LBM revealed $57.9 \pm 7.8 \mathrm{~kg}$ for men and $39.3 \pm 4.7 \mathrm{~kg}$ for women. All measurement items except for age (body weight, BMI, PBF, and LBM) showed statistically significant differences $(P<0.05)$ (Table 1).

\section{The reproducibility verification for $\mathrm{PBF}$ and $\mathrm{LBM}$ using BIA}

In order to evaluate the reproducibility of PBF and LBM using BIA, the triple repeated measurements for the same subjects were ICC-analyzed. As a result, high levels of correlation coefficients were shown in PBF $(r=0.997)$ and LBM $(r=0.998)$ (Tables 2, 3).

\section{The validity verification for $L B M$ using DEXA and BIA}

As shown in Fig. 1, the correlation between DEXA LBM and ACCUINQ BC720 LBM, ACCUINQ BC360 LBM, and ACCU-

Table 3. Reproducibility verification of LBM measured by bioelectrical impedance analyzer

\begin{tabular}{|c|c|c|c|c|c|c|}
\hline & \multicolumn{2}{|c|}{ Men $(n=40)$} & \multicolumn{2}{|c|}{ Women $(n=40)$} & \multicolumn{2}{|c|}{ Total $(n=80)$} \\
\hline & LBM (kg) & $r$ & LBM $(\mathrm{kg})$ & $r$ & LBM (kg) & $r$ \\
\hline Trial & & 0.999 & & 0.999 & & 0.998 \\
\hline $1 s t$ & $61.8 \pm 9.5$ & & $38.5 \pm 3.8$ & & $58.7 \pm 12.1$ & \\
\hline 2nd & $61.7 \pm 9.5$ & & $38.4 \pm 3.8$ & & $58.5 \pm 12.0$ & \\
\hline $3 r d$ & $61.8 \pm 9.5$ & & $38.4 \pm 3.8$ & & $58.6 \pm 12.1$ & \\
\hline
\end{tabular}

Values are presented as mean \pm standard deviation.

The intraclass correlation coefficient $(\lambda)$ were calculated from three trial measurements by the same examiner during the same test session.

LBM, lean body mass. 

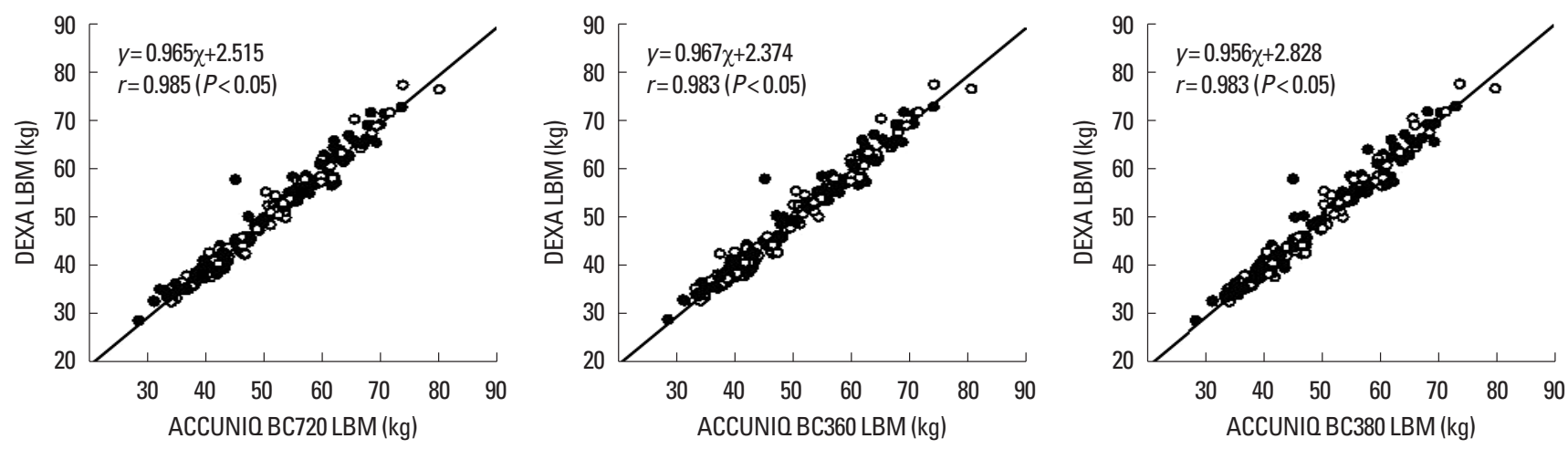

Fig. 1. Correlation coefficients and regression curve among ACCUNIQ BC720 LBM, ACCUNIQ BC360 LBM, ACCUNIQ BC380 LBM, and DEXA LBM. DEXA LBM, lean body mass by using dual energy X-ray absorptiometry; ACCUNIO BC720 LBM, lean body mass by using ACCUNIQ body composition 720; ACCUNIQ BC360 LBM, lean body mass by using ACCUNIQ body composition 360; ACCUNIQ BC380 LBM, lean body mass by using ACCUNIQ body composition 380.
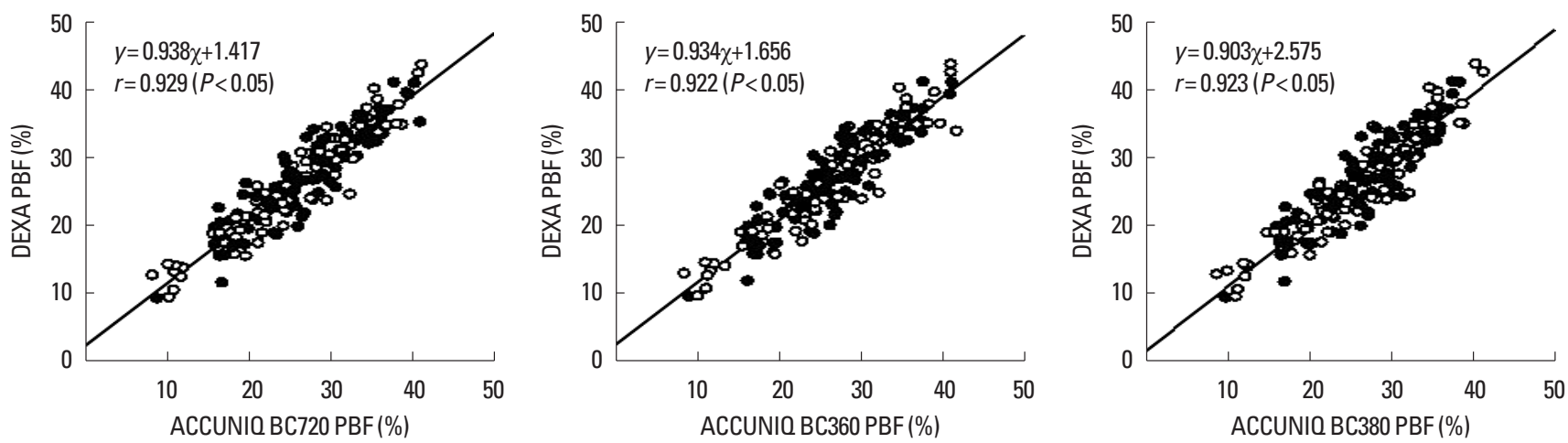

Fig. 2. Correlation coefficients and regression curve among ACCUNIQ BC720 PBF, ACCUNIQ BC360 PBF, ACCUNIQ BC380 PBF, and DEXA PBF. DEXA PBF, percent body fat by using dual energy X-ray absorptiometry; ACCUNIQ BC720 PBF, percent body fat by using ACCUNIO body composition 720; ACCUNIQ BC360 PBF, percent body fat by using ACCUNIQ body composition 360; ACCUNIQ BC380 PBF, percent body fat by using ACCUNIQ body composition 380.

INQ BC380 LBM was high with correlation coefficients of $r=0.985, r=0.983$, and $r=0.983$, respectively; the correlation with ACCUINQ BC720 LBM was the highest at $98.5 \%$, and the correlation among each group showed statistically significant differences $(P<0.05)$.

\section{The validity verification for PBF using DEXA and BIA}

As shown in Fig. 2, the correlation between DEXA PBF and ACCUINQ BC720 PBF, ACCUINQ BC360 PBF, and ACCUINQ BC380 PBF was somewhat lower than LBM with correlation coefficients of $r=0.929, r=0.922$, and $r=0.923$, respectively. Compared with preceding studies, however, the correlation is higher. Among them, the correlation with ACCUINQ BC720 PBF was the highest at $92.9 \%$, and the correlation among each group showed statistically significant differences $(P<0.05)$.
Table 4. Correlation coefficients ( $)$ among ACCUNIO BC720, ACCUNIO BC360, ACCUNIQ BC380, and DEXA in men ( $n=97)$

\begin{tabular}{lccc}
\hline Variable & $\begin{array}{c}\text { ACCUNIQ BC720 } \\
\text { LBM }\end{array}$ & $\begin{array}{c}\text { ACCUNIQ BC360 } \\
\text { LBM }\end{array}$ & $\begin{array}{c}\text { ACCUNIQ BC380 } \\
\text { LBM }\end{array}$ \\
\hline DEXA LBM $(\mathrm{kg})$ & $0.951^{*}$ & $0.950^{*}$ & $0.946^{*}$ \\
DEXA PBF $(\%)$ & $0.889^{*}$ & $0.888^{*}$ & $0.881^{*}$ \\
\hline
\end{tabular}

ACCUNIQ BC720 LBM, lean body mass by using ACCUNIQ body composition 720; ACCUNIQ BC360 LBM, lean body mass by using ACCUNIQ body composition 360; ACCUNIO BC380 LBM, lean body mass by using ACCUNIQ body composition 380; DEXA LBM, lean body mass by using dual energy $X$-ray absorptiometry; DEXA PBF, percent body fat by using dual energy $X$-ray absorptiometry.

${ }^{*} P<0.05$, significant difference.

\section{The LBM and PBF validity verification using DEXA and BIA for men}

As shown in Table 4, the correlation between DEXA LBM and ACCUINQ BC720 LBM, ACCUINQ BC360 LBM, and ACCUINQ BC380 LBM for men was high with correlation coefficients of $r=0.951, r=0.950$, and $r=0.946$, respectively; the correlation 
Table 5. Correlation coefficients ( $)$ among ACCUNIO BC720, ACCUNIO BC360, ACCUNIQ BC380, and DEXA in women $(n=103)$

\begin{tabular}{lccc}
\hline Variable & $\begin{array}{c}\text { ACCUNIQ BC720 } \\
\text { LBM }\end{array}$ & $\begin{array}{c}\text { ACCUNIO BC360 } \\
\text { LBM }\end{array}$ & $\begin{array}{c}\text { ACCUNIQ BC380 } \\
\text { LBM }\end{array}$ \\
\hline DEXA LBM $(\mathrm{kg})$ & $0.956^{*}$ & $0.946^{*}$ & $0.957^{*}$ \\
DEXA PBF $(\%)$ & $0.898^{*}$ & $0.878^{*}$ & $0.893^{*}$ \\
\hline
\end{tabular}

ACCUNIQ BC720 LBM, lean body mass by using ACCUNIQ body composition 720; ACCUNIO BC360 LBM, lean body mass by using ACCUNIQ body composition 360; ACCUNIQ BC380 LBM, lean body mass by using ACCUNIQ body composition 380; DEXA LBM, lean body mass by using dual energy $X$-ray absorptiometry; DEXA PBF, percent body fat by using dual energy $X$-ray absorptiometry.

${ }^{*} P<0.05$, significant difference.

with ACCUINQ BC720 LBM was the highest at $95.1 \%$, and the correlation among each group showed statistically significant differences $(P<0.05)$. Additionally, the correlation between DEXA PBF and ACCUINQ BC720 PBF, ACCUINQ BC360 PBF, and ACCUINQ BC380 PBF for men was somewhat lower than LBM with correlation coefficients of $r=0.889, r=0.888$, and $r=0.881$, respectively. Compared to preceding studies, however, the correlation is high. Among them, the correlation with ACCUINQ $\mathrm{BC} 720 \mathrm{PBF}$ was the highest at $88.9 \%$, and the correlation among each group showed statistically significant differences $(P<0.05)$.

\section{The LBM and PBF validity verification using DEXA and BIA for women}

As shown in Table 5, the correlation between DEXA LBM and ACCUINQ BC720 LBM, ACCUINQ BC360 LBM, and ACCUINQ BC380 LBM for women was high with correlation coefficients of $r=0.956, r=0.946$, and $r=0.957$, respectively; the correlation with ACCUINQ BC380 LBM was the highest at $95.7 \%$, and the correlation among each group showed statistically significant differences $(P<0.05)$. Additionally, the correlation between DEXA PBF and ACCUINQ BC720 PBF, ACCUINQ BC360 PBF, and ACCUINQ BC380 PBF for women was somewhat lower than LBM with correlation coefficients of $r=0.898, r=0.878$, and $r=0.893$, respectively. Compared to preceding studies, however, the correlation is high. Among them, the correlation with ACCUINQ BC720 PBF was the highest at $89.8 \%$, and the correlation among each group showed statistically significant differences $(P<0.05)$.

\section{DISCUSSION}

Despite a vast amount of research from various angles on the reliability and validity of body composition measuring devices using BIA, the algorithm of the measuring devices using BIA for improving the correlation and the reproducibility of repeated measurements for the same research subjects has been developed through references using various devices with high validity. Among them, it is known that the most widely used, accurate, reliable and valid body composition measurements are underwater weighting (UWW), nuclear magnetic resonance, CT, and DEXA. DEXA especially tests research subjects in a lying position with the three-departed compartment model that divides the human body into body fat, minerals, and residual volume, which makes it more efficient and stable than UWW, which has generally been used; when measured repeatedly, the reliability is high with an error within 1\% (WHO Expert Consultation, 2004), and it is known that DEXA has higher accuracy than UWW for both Western and Eastern people when it comes to body composition measurements (Deurenberg et al., 2001; Wang et al., 1998). Therefore, the WHO Expert Consultation (2004) recommends DEXA as the reference for research on body composition. Such tools, however, are difficult to use at various body composition-related sites as they require a lot of time, costly equipment, and professional testers.

Recently, the most widely used and useful clinical body composition measurement method is BIA. BIA predicts body composition using the difference in electrical conductivity according to the physical quality of tissues. Electrical conductivity is proportionate to water and electrolyte amount, and the rounder the cell shape, the lower the value. Fat tissues consist of round cells, and contain relatively less water than other tissues including muscles; the more the fat mass, the lower the electrical conductivity (Korean Society for the Study of Obesity, 2008). BIA automatically calculate and show the area of visceral fat through a regression equation developed by itself (Jackson et al., 1988), and has frequently been reported to be suitable for accurate measurement of fat mass (FM), PBF, and LBM (Cleary et al., 2008). Estimation of human body density using BIA is conducted under the following two premises: First, the human body is completely cylindrical with a different length and cross-sectional area. Second, the resistance when electric current passes through the human body at a fixed frequency (e.g., $50 \mathrm{kHz}$ ) is proportionate to the length of the conductor, i.e., height, and is inversely proportional to cross-sectional area (Ward and Müller, 2013).

Moreover, LBM consists of three major components with different densities (water, mineral, and protein). As changes have recently occurred in LBM density by various factors including maturation, age, race, or gender, it is likely that errors occur if the conventional equation is applied to all targets (Demura et al., 
2002). Accordingly, different estimation equations for ACCUNIQ BC720, ACCUNIQ BC360, and ACCUNIQ BC380 for athletes who have worked out a lot including weight training, obese people with a high amount of body fat, and seniors who have different muscle densities were developed to improve a new algorithm and release new products. Thus, this research verified the reproducibility of repeated measurements by body composition devices using BIA, the validity of LBM and PBF measured by DEXA, and that of LBM and PBF using the resistances of body weight and height by BIA.

As a result, the reproducibility of repeated measurements of LBM and PBF using BIA for the same people was $r=0.998$ and $r=997$, respectively; the result showed higher reliability for the repeated measurements than Healthkeeper, Inbody 320, Inbody Band, and Inbody 720 , which were suggested in preceding research (Jensky-Squires et al., 2008). In the correlation verification of LBM and PBF by three types of BIA based on the reference value by DEXA and the validity verification through regression equations, the correlation coefficients of ACCUNIQ BC720, ACCUNIQ BC360, ACCUNIQ BC380 LBM, and DEXA LBM showed positive correlations with $r=0.985, r=0.983$, and $r=0.983$. While PBF also showed positive correlations with LBM with somewhat low coefficients of $r=0.929, r=0.922$, and $r=0.923$, this research revealed significantly higher validity compared to different preceding studies. Additionally, the validity verification by gender also showed a high positive correlation. Such results revealed higher validity than those from preceding research (Pichard, 1997; Lee et al., 2001; Wang et al., 1998; Xie et al., 1999). Moreover, this research also revealed that men have greater differences in LBM or PBF compared to women when it comes to the validity range by gender. Existing BIA-based equipment showed a significantly higher correlation with FM, but had a lower correlation with most LBM due to excessively measured BIA compared to DEXA. As BIA is based on resistance measurement, it is considered that the circumference of the human body or lengths of each part of the body may have affected the measurement and prediction. This research, however, was able to measure LBM with high accuracy, which was difficult with noninvasive methods due to a significantly high correlation between the measurement by DEXA that is the basis for LBM and the measurement from the BIA-based ACCUNIQ BC. The reason for slight errors of the correlation in the measurements among products is considered to be caused by different included estimation equations according to the scale and characteristics of the research subjects and the measurement product.
As this research showed a higher correlation in terms of accuracy compared to existing BIA-based body composition measurement techniques, and the accuracy of LBM was improved with high correlation coefficients through the algorithm improved using the multi-frequency BIA method in the ACCUNIQ BC products. Therefore, they are considered suitable for objective body composition measurement for healthy and strong athletes, obese people with loss of FM, and seniors who have different muscle densities at sports rehabilitation sites.

\section{CONFLICT OF INTEREST}

No potential conflict of interest relevant to this article was reported.

\section{ACKNOWLEDGMENTS}

We sincerely thank the technical supports in SELVAS Healthcare Inc. and this study was conducted by research funds from Gwangju University in 2018.

\section{REFERENCES}

Cleary J, Daniells S, Okely AD, Batterham M, Nicholls J. Predictive validity of four bioelectrical impedance equations in determining percent fat mass in overweight and obese children. J Am Diet Assoc 2008;108: 136-139.

Demura S, Yamaji S, Goshi F, Kobayashi H, Sato S, Nagasawa Y. The validity and reliability of relative body fat estimates and the construction of new prediction equations for young Japanese adult males. J Sports Sci 2002;20:153-164.

Deurenberg P, Andreoli A, Borg P, Kukkonen-Harjula K, de Lorenzo A, van Marken Lichtenbelt WD, Testolin G, Vigano R, Vollaard N. The validity of predicted body fat percentage from body mass index and from impedance in samples of five European populations. Eur J Clin Nutr 2001;55:973-979.

Fields DA, Hunter GR, Goran MI. Validation of the BOD POD with hydrostatic weighing: influence of body clothing. Int J Obes Relat Metab Disord 2000;24:200-205.

Haarbo J, Gotfredsen A, Hassager C, Christiansen C. Validation of body composition by dual energy X-ray absorptiometry (DEXA). Clin Physiol 1991;11:331-341.

Jackson AS, Pollock ML, Graves JE, Mahar MT. Reliability and validity of bioelectrical impedance in determining body composition. J Appl Physiol (1985) 1988;64:529-534. 
Jensky-Squires NE, Dieli-Conwright CM, Rossuello A, Erceg DN, McCauley S, Schroeder ET. Validity and reliability of body composition analysers in children and adults. Br J Nutr 2008;100:859-865.

Katch FI, Hortobagyi T, Denahan T. Reliability and validity of a new method for the measurement of total body volume. Res Q Exerc Sport 1989; 60:286-291.

Kitano T, Kitano N, Inomoto T, Futatsuka M. Evaluation of body composition using dual-energy $\mathrm{X}$-ray absorptiometry, skinfold thickness and bioelectrical impedance analysis in Japanese female college students. J Nutr Sci Vitaminol (Tokyo) 2001;47:122-125.

Kohrt WM. Preliminary evidence that DEXA provides an accurate assessment of body composition. J Appl Physiol (1985) 1998;84:372-377.

Korean Society for the Study of Obesity. Evaluation of body composition. In: Korean Society for the Study of Obesity, editors. Clin obesity. 3th ed. Seoul: Korea Medicine; 2008. p. 184-186.

Lee SW, Song JH, Kim GA, Lee KJ, Kim MJ. Assessment of total body water from anthropometry-based equations using bioelectrical impedance as reference in Korean adult control and haemodialysis subjects. Nephrol Dial Transplant 2001;16:91-97.

Nakadomo F, Tanaka K, Hazama T, Maeda K. Assessment of body composition in Japanese females by bioelectrical impedance analysis. Jpn J Physic Fit Sport Med 1990;39:164-172.

Pelizzari CA, Chen GT, Spelbring DR, Weichselbaum RR, Chen CT. Accurate three-dimensional registration of $\mathrm{CT}, \mathrm{PET}$, and/or MR images of the brain. J Comput Assist Tomogr 1989;13:20-26.

Pichard C, Kyle UG, Gremion G, Gerbase M, Slosman DO. Body compo- sition by x-ray absorptiometry and bioelectrical impedance in female runners. Med Sci Sports Exerc 1997;29:1527-1534.

Saarelainen J, Rikkonen T, Honkanen R, Kröger H, Tuppurainen M, Niskanen L, Jurvelin JS. Is discordance in bone measurements affected by body composition or anthropometry? A comparative study between peripheral and central devices. J Clin Densitom 2007;10:312-318.

Stolarczyk LM, Heyward VH, Hicks VL, Baumgartner RN. Predictive accuracy of bioelectrical impedance in estimating body composition of Native American women. Am J Clin Nutr 1994;59:964-970.

Tanaka K, Nakadomo F, Watanabe K, Inagaki A, Kim HK, Matsuura Y. Body composition prediction equations based on bioelectrical impedance and anthropometric variables for Japanese obese women. Am J Hum Biol 1992;4:739-745.

Wang ZM, Deurenberg P, Guo SS, Pietrobelli A, Wang J, Pierson RN Jr, Heymsfield SB. Six-compartment body composition model: intermethod comparisons of total body fat measurement. Int J Obes Relat Metab Disord 1998;22:329-337.

Ward LC, Müller MJ. Bioelectrical impedance analysis. Eur J Clin Nutr 2013;67 Suppl 1:S1.

WHO Expert Consultation. Appropriate body-mass index for Asian populations and its implications for policy and intervention strategies. Lancet 2004;363:157-163.

Xie X, Kolthoff N, Bärenholt O, Nielsen SP. Validation of a leg-to-leg bioimpedance analysis system in assessing body composition in postmenopausal women. Int J Obes Relat Metab Disord 1999;23:1079-1084. 